\title{
Mathematical and Experimental Validation of Flux Dialysis Method: An Improved Approach to Measure Unbound Fraction for Compounds with High Protein Binding and Other Challenging Properties
}

\author{
J. Cory Kalvass, Colin Phipps, Gary J. Jenkins, Patricia Stuart, Xiaomei Zhang, Lance Heinle, \\ Marjoleen J. M. A. Nijsen, and Volker Fischer
}

Drug Metabolism, Pharmacokinetics and Bioanalysis, Research and Development, AbbVie, North Chicago, Illinois

Received October 19, 2017; accepted January 31, 2018

\begin{abstract}
A flux dialysis method to measure unbound fraction $\left(f_{u}\right)$ of compounds with high protein binding and other challenging properties was tested and validated. This method is based on the principle that the initial flux rate of a compound through a size-excluding dialysis membrane is proportional to the product of the compound initial concentration, $f_{\mathrm{u}}$, and unbound dialysis membrane permeability $\left(P_{\text {mem }}\right)$. Therefore, $f_{\mathrm{u}}$ can be determined from the initial concentration and flux rate, assuming membrane $\boldsymbol{P}_{\text {mem }}$ is known. Compound initial flux rates for 14 compounds were determined by dialyzing human plasma containing compound (donor side) versus compound-free plasma (receiver side) and measuring the rate of compound appearance into the receiver side. Eleven compounds had known $f_{\mathrm{u}}$ values obtained from conventional methods (ranging
\end{abstract}

from 0.000013 to 0.22 ); three compounds (bedaquiline, lapatinib, and pibrentasvir) had previously qualified $\boldsymbol{f}_{\mathrm{u}}$ values (e.g., <0.001). $\boldsymbol{P}_{\text {mem }}$ estimated from flux rates and known $f_{u}$ values did not meaningfully differ among the compounds and were consistent with previously published values, indicating that $\boldsymbol{P}_{\mathrm{mem}}$ is a constant for the dialysis membrane. This $\boldsymbol{P}_{\text {mem }}$ constant and the individual compound flux rates were used to calculate $f_{\mathrm{u}}$ values. The flux dialysis $f_{\mathrm{u}}$ values for the 11 compounds were in good agreement with their reported $f_{\mathrm{u}}$ values (all within 2.5 -fold; $R^{2}=0.980$ ), confirming the validity of the method. Furthermore, the flux dialysis method allowed discrete $f_{\mathrm{u}}$ to be estimated for the three compounds with previously qualified $f_{\mathrm{u}}$. Theoretical and experimental advantages of the flux dialysis method over other dialysis-based protein binding methods are discussed.

\section{Introduction}

Determination of the unbound fraction $\left(f_{\mathrm{u}}\right)$ of a compound is essential for the interpretation of disposition, efficacy (i.e., free drug exposure at the pharmacologic target), drug-drug interaction, and safety data. Although many different methods (e.g., ultrafiltration, ultracentrifugation, equilibrium gel filtration) have been used to measure compound $f_{\mathrm{u}}$ from biologic matrices, equilibrium dialysis is one of the most commonly used approaches since it is inexpensive, easy to perform, automatable, and reliable for most compounds (Di et al., 2017); however, accurate $f_{\mathrm{u}}$ values can be determined by equilibrium dialysis only when equilibrium is truly achieved, compound and matrix do not degrade during the time course of dialysis, and the buffer unbound concentration is greater than the bioanalytical lower limit of quantification (LLOQ). Several dialysis-based alternative approaches (e.g., plasma vs. plasma competition, plasma dilution, and presaturation

The design, study conduct, and financial support for this study were provided by AbbVie. AbbVie participated in the interpretation of data, writing, review, and approval of publication. All authors are current employees of AbbVie.

https://doi.org/10.1124/dmd.117.078915. methods) that attempt to address some of these challenges have been proposed and recently reviewed in the literature (Clarke et al., 2008; Di et al., 2012; Riccardi et al., 2015), but the need to reach equilibrium, low compound concentrations on the receiver side and the liabilities of nonspecific binding still exist. An infrequently used dialysis approach, dynamic dialysis, first developed in the 1950s, has the capability of measuring much lower $f_{\mathrm{u}}$ values than equilibrium dialysis and has the potential to address these challenges. Using this approach, compoundspiked serum is dialyzed against compound-free serum, and the relative $f_{\mathrm{u}}$ of the compound is determined from the initial slope of compound appearance in the receiver compartment using the principle that the compound's relative permeability across the dialysis membrane is proportional to the compound's relative $f_{\mathrm{u}}$ (Christensen, 1959). The absolute $f_{\mathrm{u}}$ of a compound can be determined in a similar manner if a compound's unbound dialysis membrane permeability $\left(P_{\text {mem }}\right)$ is known (Meyer and Guttman, 1968, 1970a,b, Ross, 1978). Measuring a compound's $f_{\mathrm{u}}$ with the dynamic dialysis method offers advantages over equilibrium dialysis, namely, allowing much higher receiver concentration to be achieved (enabling measurements of lower $f_{\mathrm{u}}$ values for a given LLOQ) and reducing nonspecific binding to the dialysis

ABBREVIATIONS: A, dialysis membrane surface area; C, compound concentration; D, matrix dilution factor; $f_{\mathrm{u}}$, unbound fraction; HPLC, highperformance liquid chromatography; $k_{\text {loss }}$, compound first-order rate of irreversible loss; $K_{n s b}$, binding potential $\left(B_{\max } / K_{D}\right)$; LLOQ, lower limit of quantification; MS/MS, tandem mass spectrometry; MWCO, molecular weight cutoff; $\mathrm{P}_{\text {mem }}$, compound unbound dialysis membrane permeability; $\mathrm{R}$, receiver/donor concentration ratio; $\mathrm{R}_{\text {eq }}$, receiver/donor concentration ratio at steady-state equilibrium; $R_{\text {slope, }}$ derivative of concentration ratio with respect to time $(\mathrm{dR} / \mathrm{dt})$ under initial sink conditions; $\mathrm{SF}$, scaling factor; $\mathrm{t}_{1 / 2 \text {,eq }}$, dialysis equilibration half-life; $V$, compartment-volume; $\mathrm{X}_{0}$, compound mass at time zero. 
device (Ross, 1978). Despite these advantages, dynamic dialysis is rarely used compared with equilibrium dialysis. During the 1950s1970s, when dynamic dialysis methods were primarily being developed and used, they likely suffered from the following disadvantages compared with equilibrium dialysis:

1. A higher level of radiochemical purity was required since dynamic dialysis methods are highly susceptible to experimental artifacts introduced by radiochemical impurities (Christensen, 1959).

2. Multiple time points needed for dynamic dialysis decreased experimental throughput and increased workload compared with single-time-point equilibrium dialysis. This was especially true considering the available technology of the time (e.g., large individual closedcell dialysis units, bioanalytical methods with long run times, often requiring extensive sample extraction or cleanup).

3. Dynamic dialysis method required measurement of $P_{\mathrm{mem}}$ for each compound, which could be laborious and challenging for compounds exhibiting high nonspecific binding (Meyer and Guttman, 1970a).

We reasoned that these disadvantages could be mitigated by modern technology (e.g., improved bioanalytical methods, mass spectrometry, high-throughput 96-well equilibrium dialysis devices, kinetic modeling) and sought to test and validate a new modern, dynamic dialysis approach (flux dialysis) as an improved way to measure $f_{\mathrm{u}}$ for compounds with high protein binding and other challenging properties. To explore the intrinsic differences between flux dialysis and other dialysis-based methods, a dialysis kinetic model was built. The model describes the time course of compound concentrations in the donor and receiver compartments during dialysis, accounting for both compound degradation and device nonspecific binding. The utility and applicability of the flux dialysis approach were further investigated by using a test set of 14 compounds with reported human plasma $f_{\mathrm{u}}$ values covering four orders of magnitude (0.000013-0.22) and diverse physicochemical properties (mol. wt. 280-1113; cLogP -0.88 to 8.1; polar-surface area 6.5-204 $\mathrm{A}^{2}$; intrinsic solubility $0.003-450 \mu \mathrm{M}$ ). Three of the test-set compounds (bedaquiline, lapatinib, and pibrentasvir) have extremely high plasma-protein binding values such that only qualified $f_{\mathrm{u}}$ values have been reported using conventional protein binding methods. Two other compounds, UCN-01 and venetoclax, were selected for inclusion in the test set because discrete $f_{\mathrm{u}}$ values could be measured only using diluted plasma; and in addition, $\mathrm{UCN}-01$ demonstrated saturable plasma-protein binding. A detailed analysis of UCN-01 and venetoclax $f_{\mathrm{u}}$ values determined from diluted plasma (using conventional and/or flux dialysis) and undiluted plasma (using flux dialysis) was conducted. Accuracy, precision, and sensitivity of $f_{\mathrm{u}}$ measurements determined from the flux dialysis method were compared with reported $f_{\mathrm{u}}$ results determined from other methods. Theoretical and experimental advantages of the flux dialysis method are discussed in the context of other dialysis-based protein binding methods.

\section{Materials and Methods}

Materials. Human plasma was purchased from Bioreclamation IVT (Hicksville, NY); typical lots consisted of a pool from 25 male donors. Test compounds (amiodarone, bedaquiline, glecaprevir, glyburide, indomethacin, imipramine, itraconazole, lapatinib, nelfinavir, pibrentasvir, quinidine, sertraline, and venetoclax) were obtained from the Global Compound Logistics and Operations Department at AbbVie, Inc (Lake County, IL). UCN-01 was obtained from Sigma-Aldrich (St. Louis, MO) (catalog no. U6508-5MG). The 96-well equilibrium dialysis device (HTD-96b, highthroughput dialysis device in a 96-well format) and cellulose membranes with molecular weight cutoff (MWCO) of 12-14 kDa were purchased from HT-Dialysis (Gales Ferry, CT). Breathe Easy gas-permeable adhesive seals were obtained from Sigma-Aldrich. Deep well plates were from Analytical Sales and Service (Flanders, NJ). Sodium azide was obtained from Sigma-Aldrich (catalog no. S2002-5G).

Dialysis Kinetic Modeling and Derivation for Flux Dialysis Method. A two-compartment kinetic model describing the time course of compound donor and receiver concentrations during dialysis was constructed (Fig. 1). The model structure was general, allowing consideration of dialysis between any two matrices (e.g., plasma vs. buffer, buffer vs. buffer, plasma vs. plasma). Kinetic relationships revealed that under initial sink conditions (i.e., donor matrix concentration $\approx$ initial concentration, and receiver unbound $<<$ donor unbound matrix concentration), the rate of compound appearance into the receiver compartment (i.e., flux) is directly proportional to the donor matrix unbound fraction $\left(f_{\mathrm{u}, \text { donor }}\right)$. Mathematical relationships between the $f_{\mathrm{u}}$ values in donor and receiver matrices $\left(f_{\mathrm{u} \text {,donor }}\right.$ and $\left.f_{\mathrm{u} \text {,receiver }}\right)$, compound unbound dialysis-membrane permeability $\left(P_{\text {mem }}\right)$, dialysis-membrane area $(\mathrm{A})$, receiver/donor compound concentration ratio $(R)$, compartment volumes $(V)$, and initial flux rate $\left(R_{\text {slope }}\right)$ were formulated. The resulting relationships provided a mathematical basis to calculate $f_{\mathrm{u} \text {,donor }}$ and $f_{\mathrm{u} \text {,receiver }}$ from receiver/donor concentration ratio data obtained from dialysis experiments. An abbreviated description of the kinetic model is presented here; however, an expanded derivation accounting for the influence of nonspecific binding and compound degradation is presented and solved in Appendix A.

The dialysis kinetic model (Fig. 1) comprises a donor matrix and a receiver matrix compartment separated by a semipermeable membrane. Assuming negligible nonspecific binding to device and negligible compound degradation, the differential equations for donor and receiver concentrations are given by eq. 1 and eq. 2 :

$$
\begin{gathered}
\mathrm{V}_{\text {donor }} \frac{\mathrm{dC}_{\text {donor }}}{\mathrm{dt}}=\left(\mathrm{P}_{\text {mem }} \times \mathrm{A}\right)\left(\mathrm{f}_{\mathrm{u}, \text { receiver }} \times \mathrm{C}_{\text {receiver }}-\mathrm{f}_{\mathrm{u}, \text { donor }} \times \mathrm{C}_{\text {donor }}\right) \\
\mathrm{V}_{\text {receiver }} \frac{\mathrm{dC}_{\text {receiver }}}{\mathrm{dt}}=\left(\mathrm{P}_{\text {mem }} \times \mathrm{A}\right)\left(\mathrm{f}_{\mathrm{u}, \text { donor }} \times \mathrm{C}_{\text {donor }}-\mathrm{f}_{\mathrm{u}, \text { receiver }} \times \mathrm{C}_{\text {receiver }}\right)
\end{gathered}
$$

The initial conditions are $C_{\mathrm{donor}}=X_{0} / V_{\text {donor }}$ and $C_{\text {receiver }}=0$, where $X_{0}$ is the amount of compound in the donor compartment at time $=0$. At equilibrium, the system reaches a steady state where $\mathrm{f}_{\mathrm{u} \text {,receiver }} \times \mathrm{C}_{\text {receiver }}=\mathrm{f}_{\mathrm{u}, \text { donor }} \times \mathrm{C}_{\mathrm{donor}}$, and

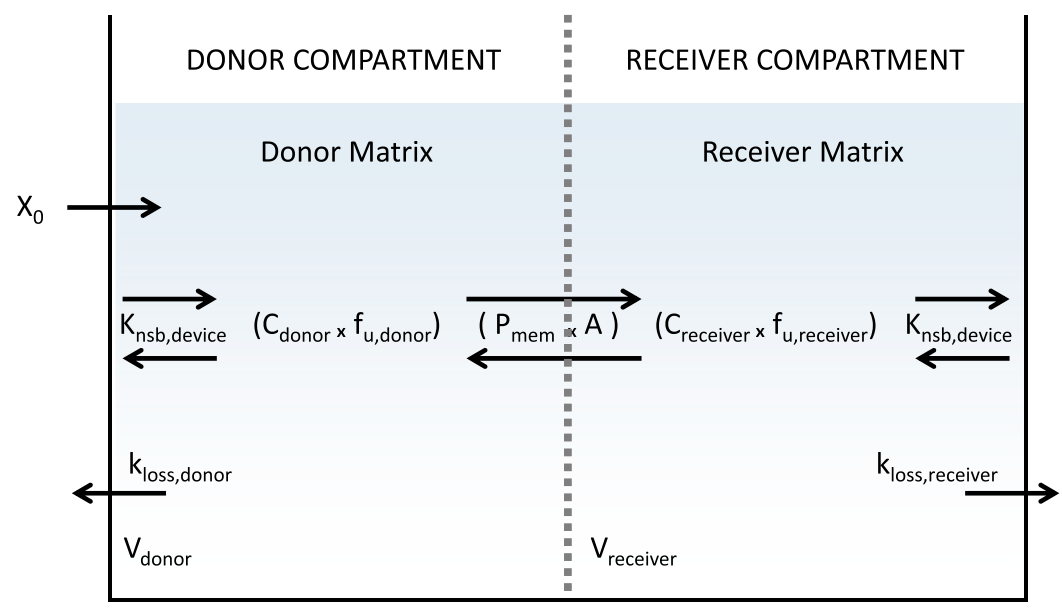

Fig. 1. Dialysis kinetic model. The model is composed of donor and receiver matrix compartments separated by a semipermeable membrane. The receiver compartment is always defined as the compartment with the lowest matrix unbound compound concentration. $X_{0}$ denotes compound mass at time zero, $V$ the compartment volumes, $A$ the membrane surface area, and $P_{\text {mem }}$ the unbound compound dialysis-membrane permeability. $C$ denotes the total compound matrix concentrations, $f_{\mathrm{u}}$ the compound matrix unbound fractions, $k_{\text {loss }}$ the compound first-order rates of irreversible loss (e.g., degradation or covalent binding), and $K_{\text {nsb,device }}$ the compound nonspecific binding potential to the dialysis device. 
therefore $\frac{\mathrm{C}_{\text {receiver }}}{\mathrm{C}_{\text {dener }}}=\frac{\mathrm{f}_{\mathrm{u} \text { donor }}}{\mathrm{f}_{\text {ureceiver }}}$. The concentration ratio as a function of time is denoted

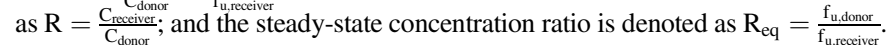

There is no loss in this model, so mass is conserved and satisfies the relationship $\left(\mathrm{C}_{\text {receiver }} \times \mathrm{V}_{\text {receiver }}\right)+\left(\mathrm{C}_{\text {donor }} \times \mathrm{V}_{\text {donor }}\right)=X_{0}$ at all times.

Under initial sink conditions, the following conditions are held:

$$
\begin{gathered}
\mathrm{V}_{\mathrm{donor}} \frac{\mathrm{dC}_{\mathrm{donor}}}{\mathrm{dt}}=0 ; \mathrm{C}_{\text {donor }}=X_{0} / \mathrm{V}_{\text {donor }} ; \text { and } \\
\mathrm{f}_{\mathrm{u}, \text { receiver }} \times \mathrm{C}_{\text {receiver }} \ll \mathrm{f}_{\mathrm{u} \text {,donor }} \times \mathrm{C}_{\text {donor }}
\end{gathered}
$$

Solving for compound flux rate into the receiver compartment under these sink conditions yields the following:

$$
\text { flux }=\mathrm{V}_{\text {receiver }} \times \frac{\mathrm{dC}_{\text {receiver }}}{\mathrm{dt}}=\mathrm{f}_{\mathrm{u}, \text { donor }} \times \mathrm{C}_{\text {donor }} \times \mathrm{P}_{\text {mem }} \times \mathrm{A}
$$

Equation 3 indicates initial rate of compound appearance into receiver compartment (i.e., flux) is directly proportional to $f_{\mathrm{u}, \text { donor }}$.

Since the donor concentration is approximately constant under initial sink conditions, the initial flux rate in eq. 3 can be rearranged to yield the approximation at time $=0$ :

$$
\frac{\mathrm{dR}}{\mathrm{dt}}=\frac{\mathrm{f}_{\mathrm{u}, \text { donor }} \times \mathrm{P}_{\mathrm{mem}} \times \mathrm{A}}{\mathrm{V}_{\text {receiver }}}
$$

From eq. 4A, the initial rate of change in receiver/donor concentration ratio under initial sink conditions is directly proportional to $f_{\mathrm{u} \text {,donor }}$. This initial slope is denoted by eq. $4 \mathrm{~B}$ :

$$
\mathrm{R}_{\text {slope }}=\frac{\mathrm{f}_{\mathrm{u}, \text { donor }} \times \mathrm{P}_{\text {mem }} \times \mathrm{A}}{\mathrm{V}_{\text {receiver }}}
$$

If these initial sink conditions are met, then $R_{\text {slope }}$ can be approximated from $R$ versus time data by linearly fitting the initial slope from one or more early time points.

Alternatively, $R_{\text {slope }}$ can be approximated from $R$ versus time data by fitting the exponential eq. 5 to the data and then solving for $R_{\text {slope }}$ according to eq. 6 , where $k$ is the exponential rate constant, and $R_{\text {eq }}$ is the steady-state concentration ratio as previously defined:

$$
\begin{gathered}
\mathrm{R}=\mathrm{R}_{\mathrm{eq}} \times\left(1-\mathrm{e}^{-\mathrm{k} \times \mathrm{t}}\right) \\
\mathrm{R}_{\text {slope }}=\mathrm{R}_{\mathrm{eq}} \times \mathrm{k}
\end{gathered}
$$

Approximating the $R_{\text {slope }}$ using exponential eqs. 5 and 6 allows data from the entire time course to be used without needing to decide which time points to include or exclude from the initial slope and simultaneously provides an estimate for $R_{\text {eq. }}$. Moreover, this approach allows $R_{\text {slope }}$ to be estimated even if early time points from the initial slope are missing.

In cases where it is warranted to estimate $R_{\text {slope }}$ from a single time point, eqs. 5 and 6 can be solve for $R_{\text {slope }}$ which gives eq. 7 :

$$
\mathrm{R}_{\text {slope }}=\frac{R_{e q} \times \ln \left(\frac{R_{e q}}{R_{e q}-R}\right)}{t}
$$

Using eq. 7 to estimate $R_{\text {slope }}$ from a single time point does not require the time point to be taken during initial sink conditions; therefore, it is more accurate and preferable to estimating $R_{\text {slope }}$ from the slope between the single time point and zero, which does require the time point to be taken during initial sink conditions. If, however, two or more time points are available, then fitting exponential eq. 5 to the data would be more accurate and preferable over using a single time point and eq. 7 since $R_{\text {slope }}$ is estimated from the totality of data rather than using a single time point.

The application of these equations and theory is described in the following. Flux dialysis data were analyzed by fitting eq. 5 to receiver/donor concentration ratio $(R)$ versus time data using Phoenix 64 Build 6.3.0.395 nonlinear regression software with $1 / \mathrm{y}$ weighting. $R_{\text {slope, }}$, which equals $\mathrm{dR} / \mathrm{dt}$ at $t=0$, was calculated from either eq. 6 or eq. 7. $R_{\mathrm{eq}}$, the equilibrium receiver/donor concentration ratio, was fixed to 1 since both receiver and donor matrix were composed of identical plasma.
If $R_{\text {slope }}$ and $R_{\text {eq }}$ are estimated from flux dialysis data and the device parameters $\mathrm{A} / \mathrm{V}_{\text {receiver }}$ and $P_{\text {mem }}$ are known, then $f_{\mathrm{u} \text {,donor }}$ and $f_{\mathrm{u} \text {,receiver }}$ can be estimated by a rearrangement of eq. $4 \mathrm{~B}$ and the definition of $\mathrm{R}_{\mathrm{eq}}$ as given below in eqs. 8 and 9:

$$
\begin{gathered}
\mathrm{f}_{\mathrm{u}, \text { donor }}=\frac{\mathrm{V}_{\text {receiver }}}{\mathrm{P}_{\text {mem }} \times \mathrm{A}} \times \mathrm{R}_{\text {slope }} \\
\mathrm{f}_{\mathrm{u} \text {,receiver }}=\frac{\mathrm{f}_{\mathrm{u} \text {,donor }}}{\mathrm{R}_{\mathrm{eq}}}
\end{gathered}
$$

Here we have assumed that $P_{\text {mem }}$ is a device-specific parameter that is constant across compounds. The validity and impact of this assumption are addressed to follow. The device-specific proportionality constant between $f \mathrm{u}_{\text {,donor }}$ and $R_{\text {slope }}$ given in eq. 8 is a scaling factor (SF) that can be used to translate between the calculated $R_{\text {slope }}$ value and the desired estimate of $f_{\mathrm{u}}$. Denoting this strictly devicedependent scaling factor by $\mathrm{SF}$, where $\mathrm{SF}=\frac{\mathrm{V}_{\text {receiver }}}{\mathrm{P}_{\text {mem }} \times \mathrm{A}}$, yields the simplified version of eq. 8:

$$
\mathrm{f}_{\mathrm{u}, \mathrm{donor}}=\mathrm{SF} \times \mathrm{R}_{\text {slope }}
$$

If A/ $\mathrm{V}_{\text {receiver }}$ or $P_{\text {mem }}$ of the dialysis device is unknown, then SF must first be estimated before using eq. 10 . In this case, SF can be determined by the $R_{\text {slope }}$ and $f_{\mathrm{u}}$ from one or more reference compounds, denoted by $R_{\text {slope,ref }}$ and $f_{\mathrm{u}, \text { ref }}$, respectively. Rearranging eq. 10 for a reference compound gives eq. 11:

$$
S F=\frac{\mathrm{f}_{\mathrm{u}, \mathrm{ref}}}{R_{\text {slope }, \text { ref }}},
$$

indicating that the ratio of $f_{\mathrm{u}}$ to $R_{\text {slope }}$ is constant and equal to SF. SF can then be estimated from this ratio in eq. 11 for one reference compound or from the average $\mathrm{SF}$ values calculated for multiple reference compounds. The latter approach could reduce experimental variability or error in determining SF from a single compound $R_{\text {slope }}$ and $f_{\text {u,ref }}$ value. This estimated SF, along with a measured value of $R_{\text {slope }}$, can then be used in eq. 10 to yield specific estimates of $f_{\mathrm{u} \text {,donor }}$.

For the dialysis device used in this study, the membrane surface area-to-volume ratio $\left(\mathrm{A} / \mathrm{V}_{\text {receiver}}\right)$ provided by the manufacturer was $3.7 \mathrm{~cm}^{2} / \mathrm{ml}$ (Banker et al., 2003), and the $P_{\text {mem }}$ constant was unknown. The SF value was calculated for each reference compound, and the corresponding $P_{\text {mem }}$ was calculated from a rearrangement of the definition of SF:

$$
\mathrm{P}_{\mathrm{mem}}=\frac{1}{\mathrm{SF}} \times \frac{\mathrm{V}_{\text {receiver }}}{\mathrm{A}}=\frac{1}{\mathrm{SF}} \times \frac{1}{3.7 \mathrm{~cm}^{2} / \mathrm{mL}}
$$

The average $P_{\text {mem }}$ value constant was $75.2 \times 10^{-6} \mathrm{~cm} / \mathrm{s}$. Given the device $\mathrm{A} / \mathrm{V}_{\text {receiver }}$ ratio $\left(3.7 \mathrm{~cm}^{2} / \mathrm{ml}\right)$, this yields an equivalent value of $\mathrm{SF}=3590$ seconds. Flux dialysis $f_{\mathrm{u}}$ was calculated according to eq. 8 using this SF value, and each compound's average $R_{\text {slope }}$, giving the following relationship:

$$
\mathrm{f}_{\mathrm{u}, \text { donor }}=(3590 \mathrm{~s}) \times \mathrm{R}_{\text {slope }}
$$

$F_{\mathrm{u}}$ values from diluted plasma $\left(f_{\mathrm{u}, \text { measured }}\right)$ were converted to undiluted $f_{\mathrm{u}}$ estimates using eq. 14 :

$$
\text { Undiluted } f_{u}=\frac{1 / D}{\left(1 / f_{u, \text { measured }}-1\right)+1 / D},
$$

where $D$ is the plasma dilution factor (Kalvass and Maurer, 2002).

Compound recovery from dialysis device was calculated from eq. 15:

$$
\text { Recovery }(\%)=\frac{\mathrm{C}_{\mathrm{donor}}+\mathrm{C}_{\text {reciever }}}{\mathrm{C}_{\mathrm{donor}} \text { at time equal zero }}
$$

UCN-01 two-site binding parameters (one-site saturable, one-site nonsaturable) were calculated from flux dialysis $f_{\mathrm{u}}$ values determined at different UCN-01 concentrations (C) and plasma dilutions (D) according to eq. 16:

$$
f_{u}=\frac{C-\left(\frac{B_{m a x}}{D}+K_{D}\left(1+\frac{K_{n b b}}{D}\right)\right)+\sqrt{\left(C-\left(\frac{B_{m a x}}{D}+K_{D}\left(1+\frac{K_{n b b}}{D}\right)\right)\right)^{2}+4 K_{D}\left(1+\frac{K_{n b}}{D}\right) C}}{2\left(1+\frac{K_{n b}}{D}\right) C},
$$

where $B_{\max }$ is the saturable binding-site total concentration, $K_{\mathrm{D}}$ is the saturable binding-site equilibrium dissociation constant, $K_{\mathrm{nsb}}$ is the nonsaturable binding-site 
binding potential $\left(B_{\max , \mathrm{NS}} / K_{\mathrm{D}, \mathrm{NS}}\right)$, and $D$ is the matrix dilution factor. Derivation of this two-site saturable binding equation (one-site saturable, one-site nonsaturable) is presented in Appendix B.

Flux Dialysis Experiment. Flux dialysis experiments were conducted using a modification of a generalized equilibrium dialysis method from the literature (Kalvass et al., 2007). Regenerated cellulose membrane preparation (MWCO 12$14 \mathrm{KDa}$ ) and 96-well HTDialysis apparatus setup were consistent with the manufacturer's recommendations. Human plasma was supplemented with $10 \mu \mathrm{M}$ sodium azide (final concentration) before use to prevent microbial growth over the long incubation period. Plasma containing $1 \mu \mathrm{M}$ of test compound (donor matrix) was dialyzed against compound-free human plasma (receiver matrix) in the HTDialysis apparatus at $37^{\circ} \mathrm{C}$ in a $5 \% \mathrm{CO}_{2}$ atmosphere with shaking at $150 \mathrm{rpm}$ for up to 120 hours. Aliquots $(10 \mu \mathrm{l})$ of both receiver and donor matrix were taken at approximately $0,0.25,0.5,2,4,24,48,96,120$ hours. Aliquots were frozen for a minimum of 24 hours before analysis. Standard curve samples were prepared in human plasma containing $10 \mu \mathrm{M}$ sodium azide to match dialysis samples. Additional flux dialysis studies using the same method were conducted with UCN-01 at $0.3,1,2,3,10,30$, and $100 \mu \mathrm{M}$ in undiluted plasma and $2 \mu \mathrm{M}$ in plasma diluted 5-, 10-, and 20-fold.

Dilution-Method Equilibrium Dialysis Experiment for Venetoclax. The unbound fraction of venetoclax in human plasma was determined by equilibrium dialysis using a 96-well HTDialysis apparatus with dialysis membrane strips (MWCO 12-14 kDa). Venetoclax in 1\% plasma was equilibrated against dialyzed phosphate buffer (50 mM, pH 7.4). Dialyzed buffer was prepared by dialyzing naïve human plasma with phosphate buffer $(50 \mathrm{mM}, \mathrm{pH} 7.4)$ for 4 hours at $37^{\circ} \mathrm{C}$, mimicking a standard incubation but without the compound. Dialyzed buffer was prepared in advance and stored at $-20^{\circ} \mathrm{C}$ until use. One-percent dialyzed buffer was used on the receiver side with venetoclax in $1 \%$ plasma on the donor side. Dialyzed phosphate buffer was used during dialysis because low-molecularweight plasma components (e.g., lipids, small peptides, nutrients) are preequilibrated with receiver buffer and are expected to help keep venetoclax in solution. After incubation (4 hours, $\left.37^{\circ} \mathrm{C}\right)$, plasma $(5 \mu \mathrm{l})$ and buffer $(50 \mu \mathrm{l})$ were sampled from the dialysis plate and combined with acetonitrile/methanol containing $50 \mathrm{nM}$ carbutamide as the internal standard (quench solution). The plasma samples were matrix-blanked with buffer, and the buffer samples were matrix blanked with plasma. Likewise, standard curve samples were matrixblanked to contain identical matrix composition as the dialysis samples. Samples were vortex-mixed and stored at $4^{\circ} \mathrm{C}$, if necessary, before centrifugation and analysis by high-performance liquid chromatography (HPLC)-tandem mass spectrometry (MS/MS). The volumes of initial sampling (e.g., $5 \mu$ l plasma and $50 \mu \mathrm{l}$ buffer) were taken into account during the quantitation of venetoclax, and $f_{\mathrm{u}}$ values from diluted plasma ( $f_{\mathrm{u} \text {,measured }}$ ) were mathematically converted to values representing $f_{\mathrm{u}}$ in undiluted plasma using eq. 14, where $D$ is the plasma dilution factor (Kalvass and Maurer, 2002)

Flux Dialysis Sample Bioanalysis and Quantitation. Compound concentration in the matrix receiver and donor samples (e.g., human plasma) from the flux dialysis time course was quantitated using HPLC-MS/MS. Samples were quenched with a minimum of three volume equivalents of 95:5 acetonitrile/ methanol containing $50 \mathrm{nM}$ carbutamide (quench solution). Samples were vortexmixed and centrifuged, and supernatant was injected for LC-MS/MS analysis. The HPLC-MS/MS method is summarized as follows. Ten microliters of sample was injected onto a Fortis Pace C18 column using a CTC PAL autosampler connected to an Agilent 1290 HPLC and an AB Sciex API-5500 mass spectrometer with a turbo spray ion source. The sample was initially held at $95 \%$ of $0.1 \%$ formic acid (mobile phase A) and $5 \%$ of $0.1 \%$ formic acid in acetonitrile (mobile phase B) for 0.3 minutes. The gradient was ramped to $2 \%$ mobile phase A over the following 0.3 minutes, where it was held for an additional 0.4 minutes. The gradient was then brought back to initial conditions for 0.15 minutes. The total flow rate was held at $800 \mu \mathrm{l} / \mathrm{min}$. The specific MS/MS setting used for each compound is listed in Table 1.

Peak areas for the compounds of interest were determined with Sciex Analyst 1.6 software. The mass spectrometer response for each sample containing the test article was compared with the mass spectrometer response from a set of standard concentrations prepared for the test article using the Sciex Analyst 1.6 software. The concentration of each sample was then determined using the standard curves prepared in human plasma. The standard curves were fit using a quadratic $1 / \times$ weighted regression and met a minimum $r$-squared value of 0.98 . The LLOQ of quantitation was $1 \mathrm{nM}$ for amiodarone, bedaquiline, glyburide, and lapatinib; 2 nM for imipramine, itraconazole, pibrentasvir, sertraline, UCN01, and venetoclax; $10 \mathrm{nM}$ for glecaprevir; and $20 \mathrm{nM}$ for indomethacin, nelfinavir, and quinidine.

\section{Results}

Dialysis Modeling Results. Relationships for the dialysis $C_{\text {receiver }} /$ $C_{\text {donor }}$ time course were solved for the model structure as shown in Fig. 1. Relationships for traditional equilibrium dialysis (matrix dialyzed against buffer) indicated that nonspecific binding to device (i.e., $K_{\text {nsb,device }}$ ) has no impact on accurate $f_{\mathrm{u}}$ determination as long as equilibrium has been achieved and $k_{\text {loss }}$ is identical (or negligible) between matrix and buffer. In contrast, results for flux dialysis indicated that obtainment of equilibrium is not required, and $K_{\mathrm{nsb} \text {,device }}$ and $k_{\text {loss }}$ have no influence on accurate $f_{\mathrm{u}}$ determination as long as binding affinity to the receiver matrix is greater than the nonspecific binding to device (i.e., $f_{\mathrm{u}, \text { receiver }}<<$ $1 / \mathrm{K}_{\mathrm{nsb}, \text { device}}$ ) and $k_{\text {loss }}$ is identical (or negligible) between donor and receiver matrix, which is the case if identical matrix is used.

Dialysis equilibration half-life $\left(t_{1 / 2, \mathrm{eq}}\right)$, or time required to achieve $50 \%$ steady-state equilibrium between donor and receiver concentrations, for both flux dialysis and traditional equilibration dialysis conditions, were solved (see Appendix A). Under flux dialysis conditions, assuming identical matrix composition on both sides and negligible device nonspecific binding, $t_{1 / 2 \text {,eq }}$ is inversely proportional to $f_{\mathrm{u}}$. In contrast, under traditional equilibrium dialysis conditions, assuming negligible device nonspecific binding, $t_{1 / 2 \text {,eq }}$ is independent of $f_{\mathrm{u}}$. The dialysis time to achieve equilibrium within the HTD-96b dialysis device was calculated as $\sim 3.5$ hours using derived relationship for $t_{1 / 2 \text {,eq }}$ and assuming equilibration is achieved within $\sim 5$ equilibration half-lives (see Appendix A). In cases where device nonspecific binding is not negligible, assuming traditional equilibrium dialysis conditions, the time to reach equilibrium increases proportionally by the degree of nonspecific binding or by a factor of $K_{\text {nsb,device }}+1$.

The lowest measurable discrete $f_{\mathrm{u}}$ values from traditional equilibration dialysis (donor matrix dialyzed against buffer) and flux dialysis (identical donor and receiver matrix) were solved for as a function of bioanalytical sensitivity (i.e., LLOQ):

$$
\begin{gathered}
\text { equilibrium dialysis lowest discrete } f u=\sim \frac{\text { LLOQ }}{\mathrm{C}_{\mathrm{donor}}} \\
\text { flux dialysis lowest discrete } \mathrm{fu}=\sim \frac{\mathrm{LLOQ}}{\mathrm{C}_{\mathrm{donor}}} \times \frac{V}{P_{\text {mem }} \times A} \times \frac{1}{t}
\end{gathered}
$$

The lowest discrete $f_{\mathrm{u}}$ from the flux dialysis method, substituting the $P_{\text {mem }}$ constant $\left(75.2 \times 10^{-6} \mathrm{~cm} / \mathrm{s}\right)$ and HTDialysis A/V ratio $\left(3.7 \mathrm{~cm}^{2} / \mathrm{ml}\right)$,

TABLE 1

MS/MS instrument setting

\begin{tabular}{lcrcc}
\hline Compound & MRM & DP & CE & Ionization Mode \\
\hline Amiodarone & $646 \rightarrow 100$ & 94 & 50 & Positive \\
Bedaquiline & $555 \rightarrow 58$ & 50 & 75 & Positive \\
Glecaprevir & $840 \rightarrow 684$ & 100 & 35 & Positive \\
Glyburide & $494 \rightarrow 369$ & 54 & 22 & Positive \\
Indomethacin & $358 \rightarrow 139$ & 59 & 37 & Positive \\
Imipramine & $281 \rightarrow 86$ & 40 & 25 & Positive \\
Itraconazole & $705 \rightarrow 392$ & 83 & 52 & Positive \\
Lapatinib & $581 \rightarrow 365$ & 107 & 53 & Positive \\
Nelfinavir & $569 \rightarrow 330$ & 59 & 48 & Positive \\
Pibrentasvir & $557^{a} \rightarrow 146$ & 200 & 30 & Positive \\
Quinidine & $325 \rightarrow 172$ & 86 & 50 & Positive \\
Sertraline & $306 \rightarrow 159$ & 63 & 41 & Positive \\
7-hydroxystaurosporine (UCN-01) & $483 \rightarrow 130$ & 100 & 25 & Positive \\
Venetoclax & $869 \rightarrow 636$ & 20 & 40 & Positive \\
\hline
\end{tabular}

CE, collision energy; DP, declustering potential; MRM, multiple reaction monitoring. ${ }^{a}$ Doubled charged ion. 
TABLE 2

Advantages and disadvantages of determining unbound fraction by different dialysis methods

$(+)$ and $(-)$ symbols indicated attributes of relative advantage or disadvantage, respectively.

\begin{tabular}{|c|c|c|c|c|}
\hline & $\begin{array}{l}\text { Eq. Dialysis Method } \\
\text { (Matrix vs. Buffer) }\end{array}$ & $\begin{array}{l}\text { Eq. Dilution Method } \\
\text { (Diluted-Matrix vs. Buffer) }\end{array}$ & Eq. Competition Method (Matrix 1 vs. Matrix 2 ) & $\begin{array}{l}\text { Flux Dialysis Method } \\
\text { (Matrix vs. Matrix) }\end{array}$ \\
\hline Minimal dialysis time & $\begin{array}{l}\text { (-) Establishment of } \\
\text { equilibrium, } \\
\text { (HTDialysis } \leq 4 \mathrm{~h} \text { assuming } \\
\text { minimal nonspecific binding) }\end{array}$ & $\begin{array}{l}\text { (-) Establishment of } \\
\text { equilibrium, } \\
\text { (HTDialysis } \leq 4 \mathrm{~h} \text { assuming } \\
\text { minimal nonspecific binding) }\end{array}$ & $\begin{array}{l}\text { (-) Establishment equilibrium, (hours, } \\
\text { days, weeks, or months depending } \\
\text { on } f_{\mathrm{u}} \text { ) }\end{array}$ & $\begin{array}{l}\text { (+) Receiver conc }>>\text { LLOQ, } \\
\text { (always faster than } \\
\text { equilibrium dialysis } \\
\text { assuming same LLOQ }\end{array}$ \\
\hline $\begin{array}{l}\text { Impact of device } \\
\text { nonspecific binding }\end{array}$ & $\begin{array}{l}(-) \text { Increased time to } \\
\text { equilibrium, (although no } \\
\text { impact on } f_{\mathrm{u}} \text { ) }\end{array}$ & $\begin{array}{l}(-) \text { Increased time to } \\
\text { equilibrium (although no } \\
\text { impact on } f_{\mathrm{u}} \text { ) }\end{array}$ & $(+)$ Nonspecific binding is minimized & $\begin{array}{l}(+) \text { Nonspecific binding is } \\
\text { minimized }\end{array}$ \\
\hline $\begin{array}{l}\text { Impact of compound } \\
\text { matrix instability }\end{array}$ & $(-)$ Invalid $f_{\mathrm{u}}$ & $(-)$ Invalid $f_{\mathrm{u}}$ & $\begin{array}{l}(-) \text { Invalid } f_{\mathrm{u}} \text { if degradation rate is } \\
\text { different between matrix }{ }_{1} \text { and } \\
\text { matrix }_{2}\end{array}$ & $(+)$ No impact on $f_{\mathrm{u}}$ \\
\hline Sample composition & $\begin{array}{l}\text { (-) Matrix and buffer, two } \\
\text { standard curves or sample } \\
\text { blanking/matching require; } \\
\text { Additional GLP validation } \\
\text { and lower LLOQ may be } \\
\text { required }\end{array}$ & $\begin{array}{l}\text { (-) Matrix and buffer, two } \\
\text { standard curves or sample } \\
\text { blanking/matching require; } \\
\text { additional GLP validation } \\
\text { and lower LLOQ may be } \\
\text { required }\end{array}$ & $\begin{array}{l}\text { (-) } \text { Matrix }_{1} \text { and } \text { matrix }_{2} \text {, two standard } \\
\text { curves or sample blanking/matching } \\
\text { require; Additional GLP validation } \\
\text { may be required }\end{array}$ & $\begin{array}{l}\text { (+) All samples identical } \\
\text { matrix, single standard curve; } \\
\text { preexisting GLP assay likely } \\
\text { can be used }\end{array}$ \\
\hline LLOQ requirement & $(-)$ LLOQ $\leq$ free buffer & $\begin{array}{l}(-) \text { LLOQ } \leq \text { free buffer, } \\
\text { although free buffer increases } \\
\text { roughly proportional with } \\
\text { dilution factor }\end{array}$ & $\begin{array}{l}(+) \text { LLOQ } \leq \text { initial matrix conc } \\
\text { assuming similar } f_{\mathrm{u}} \text { between matrix } 1 \\
\text { and matrix } 2\end{array}$ & $\begin{array}{l}(+) \text { LLOQ } \leq 1 / 2 \text { initial donor } \\
\text { concentration }\end{array}$ \\
\hline $\begin{array}{l}\text { Additional advantage/ } \\
\text { disadvantages }\end{array}$ & $\begin{array}{l}\text { (+) Most commonly used } \\
\text { dialysis method }\end{array}$ & $\begin{array}{l}(-) \text { Risk for saturating protein } \\
\text { binding; }(-) \text { dilution does } \\
\text { not reduce equilibrium time }\end{array}$ & $\begin{array}{l}(-) \text { Discrete } f_{\mathrm{u}} \text { not determined, only } f_{\mathrm{u}} \\
\text { ratio between matrix } 1 \text { and matrix } 2\end{array}$ & $\begin{array}{l}\text { (-) Unconventional time } \\
\text { point(s) and data may require } \\
\text { nonlinear curve fitting }\end{array}$ \\
\hline
\end{tabular}

GLP, Good Laboratory Practice.

was calculated as $\mathrm{LLOQ} /\left(C_{\text {donor }} \times \mathrm{t}\right)$ where $t$ is expressed in hours. Comparison of nonspecific binding, compound degradation, minimal dialysis time, and LLOQ considerations across four different dialysisbased protein binding approaches are summarized in Table 2 .

Flux Dialysis Results. Using the flux dialysis method, all 14 compound receiver concentrations were above bioanalytical LLOQ at one or more time points, allowing discrete $R_{\text {slope }}$ values to be determined for each compound (Table 3). In contrast, the LLOQs achieved using AbbVie's default discovery bioanalytical method would have been insufficient to determine buffer concentrations for 8 of the 14 compounds from traditional equilibrium dialysis (plasma dialyzed against buffer), assuming $100 \%$ recovery. The flux dialysis receiver/donor matrix concentration ratio $(R)$ versus time data were adequately described by $R=R_{\mathrm{eq}} \cdot\left(1-\mathrm{e}^{-\mathrm{k} \bullet \mathrm{t}}\right)$ equation (Fig. 2). $R_{\text {slope }}$ values differed by $>10,000-$ fold among compounds (Table 3 ). $R_{\text {slope }}$ estimates were obtained with a median interday \%CV (95\%ile range) of $13 \%(2 \%-32 \%)$ (Table 3$)$.

The average $P_{\text {mem }}$ constant ( $95 \%$ prediction interval) estimated from 11 compounds with known $f_{\mathrm{u}}$ was $75.2(32-150) \times 10^{-6} \mathrm{~cm} / \mathrm{s}$. All individual compound $P_{\text {mem }}$ values were within 2.5 -fold of the average value, and no correlation between individual compound $P_{\text {mem }}$ values and

TABLE 3

$R_{\text {slope }}$, unbound fraction from flux dialysis, and unbound fraction reference values

\begin{tabular}{lccc}
\hline \multicolumn{1}{c}{ Compound } & $R_{\text {slope }}(\% \mathrm{CV}) s^{-1} \times 10^{-6}$ & Flux Dialysis $f_{\mathrm{u}}{ }^{a}$ & Reported $f_{\mathrm{u}}$ (Reference) \\
\hline Imipramine & & & 0.13 (Berry et al., 2011) \\
Quinidine & $38.9(23 \%)$ & 0.14 & 0.22 (Fremstad et al., 1979) \\
Sertraline & $31.1(8 \%)^{b}$ & 0.11 & 0.016 (Ronfeld et al., 1997) \\
Glecaprevir & $9.72(8 \%)^{b}$ & 0.035 & 0.025 (AbbVie data on file) \\
Indomethacin & $6.67(21 \%)$ & 0.024 & 0.010 (Indomethacin, 2016) \\
Nelfinavir & $2.61(13 \%)$ & 0.0094 & 0.0042 (Motoya et al., 2006) \\
Itraconazole & $0.778(7 \%)^{b}$ & 0.0028 & 0.0021 (Riccardi et al., 2015) \\
Glyburide & $0.583(5 \%)$ & 0.0021 & 0.0010 (Wan and Rehngren, 2006) \\
Lapatinib & $0.317(6 \%)$ & 0.0011 & $<0.011$ (U.S. FDA, 2007) \\
UCN-01 & $0.211(10 \%)$ & 0.00076 & $0.000073^{c}$ (Fuse et al., 1998) \\
Pibrentasvir & $0.0247(1 \%)$ & 0.000089 & $<0.0001$ (AbbVie data on file) \\
Amiodarone & $0.0172(33 \%)$ & 0.000062 & 0.00014 (Riccardi et al., 2015) \\
Bedaquiline & $0.0158(8 \%)$ & 0.000057 & $<0.0001$ (U.S. FDA, 2012) \\
Venetoclax & $0.00806(15 \%)$ & 0.000029 & 0.000013 (AbbVie data on file) \\
\hline
\end{tabular}

$\% \mathrm{CV}$, interassay \% CV calculated from experiments conducted on separated days ( $n=2-5$ experiments); \%CV values for flux dialysis $f_{u}$ are identical to $\mathrm{R}_{\text {slope }} \% \mathrm{CV}$ and therefore are not reported separately.

${ }^{a}$ Calculated as $R_{\text {slope }} \times 3590 \mathrm{~s}$.

${ }^{b}$ Intra-assay $\% \mathrm{CV}$ calculated from a single experiment run in triplicate

${ }^{c} f_{\mathrm{u}}$ value calculated from published $B_{\max }$ and $K_{\mathrm{D}}$ values (i.e., $\left.\mathrm{f}_{\mathrm{u}}=K_{\mathrm{D}} /\left(K_{\mathrm{D}}+B_{\max }\right)=0.0012 /(0.0012+16.4)=0.000073\right)$. 


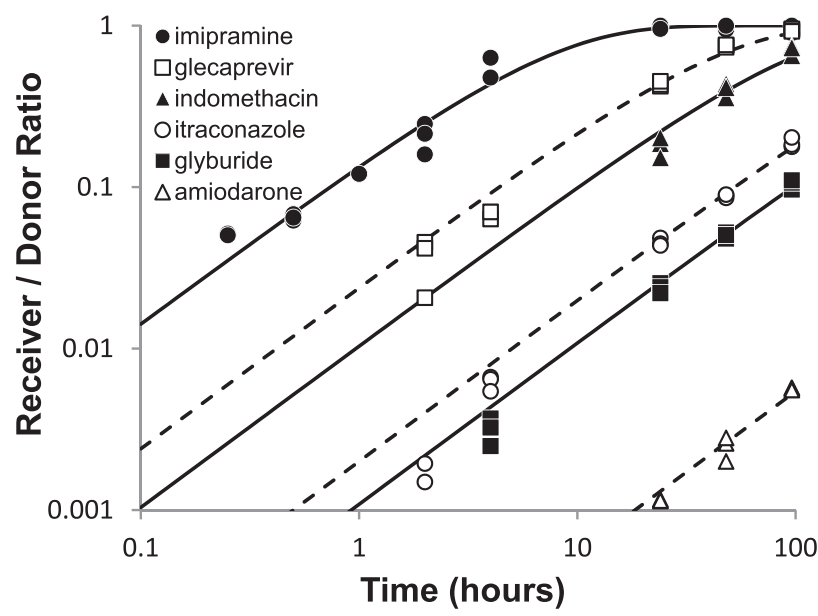

Fig. 2. Representative receiver/donor ratio versus time data from a single flux dialysis experiment. Symbols represent compound receiver/donor matrix concentration ratio determined in triplicate at different time points over a 96-hour dialysis period. Lines represent nonlinear fit of $R=R_{\mathrm{eq}} \cdot\left(1-\mathrm{e}^{-\mathrm{k} \cdot \mathrm{t}}\right)$ to receiver/donor concentration vs. time data. During the dialysis period, purchased human plasma spiked with $1 \mu \mathrm{M}$ compound (donor matrix) was dialyzed against compound-free purchased human plasma (receiver matrix). Aliquots of donor and receiver matrix $(10 \mu \mathrm{l})$ were sampled from each dialysis well at $0.25,0.5,2,4,24,48$, and 96 hours and compound concentration determined by HPLC-MS/MS.

physiochemical properties (Table 4) was found, indicating that any inaccuracy introduced by the assumption of constant $P_{\text {mem }}$ is limited to this fold-error, which is within tolerated limits of in vitro assay variability (Table 2).

Discrete $f_{\mathrm{u}}$ values from undiluted plasma and $1 \mu \mathrm{M}$ compound concentration were measurable for all 14 compounds using individual compound $R_{\text {slope }}$ values and average $P_{\text {mem }}$ constant. Flux dialysis $f_{\mathrm{u}}$ values differed more than 10,000-fold among compounds $(0.14$ $0.000012)$. Flux dialysis $f_{\mathrm{u}}$ values were highly correlated with $\left(r^{2}=\right.$ 0.980 ) and in good agreement (within \pm 2.5 -fold) with cited literature values obtained from other methods (Fig. 3). All cited literature and flux dialysis $f_{\mathrm{u}}$ values were also similar (i.e., discrete $f_{\mathrm{u}}$ values within 2-fold) to AbbVie historical equilibrium dialysis $f_{\mathrm{u}}$ values (data not shown; AbbVie historical $f_{\mathrm{u}}$ values were discrete for all compounds except lapatinib, UCN-01, pibrentasvir, amiodarone, and bedaquiline, for which only qualified $f_{\mathrm{u}}$ values could be determined).

Flux dialysis recovery for all compounds was $\geq 90 \%$, except indomethacin, which declined monoexponentially over time to $\sim 20 \%$ by 120 hours (Fig. 4A). Despite recovery of $\sim 20 \%$, the indomethacin flux time course was fitted well by eq. 5 (Fig. 4B), and individual $R_{\text {slope }}$ values estimated at each time point using eq. 7 were similar to those of $R_{\text {slope }}$ values estimated from fitting the entire time course (Fig. 4C). Indomethacin recovery was also $\sim 20 \%$ from indomethacin plasma control experiments incubated in $1.2 \mathrm{ml}$ polypropylene tubes under identical conditions as the flux dialysis experiment. Indomethacin in vitro plasma $t_{1 / 2}$ estimated from fitting the exponential equation to the recovery time course was $57 \pm 7$ hours (Fig. 4A).

UCN-01 Flux Dialysis Results. UCN-01 $f_{\mathrm{u}}$ values showed concentration-dependent changes consistent with saturable protein binding (Fig. 5). A novel mechanistic two-site saturable binding equation (one-site saturable, one-site nonsaturable) was developed and fitted simultaneously to all $\mathrm{UCN}-01$ flux dialysis $f_{\mathrm{u}}$ values generated under various experimental conditions. Estimates of $B_{\max }, K_{\mathrm{D}}$, and $K_{\mathrm{nsb}}$ obtained from fitting two-site saturable binding equation were 10.1, $0.00085 \mu \mathrm{M}$, and 21.2 , respectively, and matched closely (within 1.6fold) those previously reported in the literature $(16.4,0.0012 \mu \mathrm{M}$, and 20 , respectively) determined using an ultracentrifugation method to measure UCN-01 binding to human plasma and $\alpha$-1-acid glycoprotein (Fuse et al., 1998).

$\mathrm{UCN}-01 f_{\mathrm{u}}$ values obtained at $2 \mu \mathrm{M}$ concentration using 5-, 10-, and 20 -fold diluted plasma exceeded the observed $2 \mu \mathrm{M} f_{\mathrm{u}}$ value obtained from undiluted plasma by $\sim 110-, \sim 1300$-, and $\sim 2000$-fold, respectively (Fig. 5A). Similarly, after correcting these $f_{\mathrm{u}}$ values for plasma dilution by eq. 14 , the $f_{\mathrm{u}}$ values obtained using diluted plasma still exceeded the observed $2 \mu \mathrm{M}$ undiluted plasma value by $\sim 20$ - to $\sim 120$ fold (Fig. 5B). When the effects of plasma dilution on the $C_{\mathrm{donor}} / B_{\max }$ ratio were accounted for by multiplying $\mathrm{UCN}-01$ nominal concentrations by the plasma dilution factor (e.g., $2 \mu \mathrm{M}$ in 5-fold diluted plasma = $10 \mu \mathrm{M})$, the dilution corrected $f_{\mathrm{u}}$ values obtained from 5-, 10-, and 20 -fold diluted plasma were consistent with the expected values determined from UCN-01 plasma binding parameters (Fig. 5C).

Venetoclax Dilution-Method Equilibrium Dialysis Results. Venetoclax plasma $f_{\mathrm{u}}$ value could not be determined using undiluted

TABLE 4

Compound unbound dialysis membrane permeability $\left(P_{\text {mem }}\right)$ and physiochemical properties

Physiochemical properties calculated using Advanced Chemistry Development (ACD/Laboratories) Software V11.02 ( 1994-2017 ACD/Laboratories).

\begin{tabular}{|c|c|c|c|c|c|c|c|c|}
\hline Compound & $\begin{array}{l}P_{\text {mem }}(\% \mathrm{CV}) \\
\mathrm{cm} / \mathrm{s} \times 10^{-6}\end{array}$ & Molecular Weight & pKa (Most Acidic) & pKa (Most Basic) & $\begin{array}{c}\text { Molar Intrinsic Solubility } \\
\qquad \mu M\end{array}$ & $\log \mathrm{P}$ & $\log \mathrm{D} 7.0$ & $\begin{array}{c}\text { Polar Surface Area } \\
A^{2}\end{array}$ \\
\hline Amiodarone & & 645.31 & & 9.37 & & 7.815 & 5.51 & \\
\hline & $30.5(8 \%)$ & & & & 0.076 & & & 42.7 \\
\hline Bedaquiline & n.c. & 555.5 & 13.05 & 9.31 & 0.032 & 7.712 & 5.8 & 45.6 \\
\hline Glecaprevir & $72.1(21 \%)$ & 838.87 & 4.46 & -1.38 & 3.5 & -0.88 & -0.7 & 204 \\
\hline Glyburide & $85.7(6 \%)$ & 494 & 5.11 & -1.63 & 7.9 & 3.076 & 1.22 & 122 \\
\hline Imipramine & $80.9(23 \%)$ & 280.41 & & 9.49 & 45 & 4.355 & 2 & 6.48 \\
\hline Indomethacin & $70.5(13 \%)$ & 357.79 & 3.96 & & 17 & 4.251 & 1.29 & 68.5 \\
\hline Itraconazole & $75.0(5 \%)$ & 705.63 & & 6.47 & 0.14 & 4.995 & 4.86 & 101 \\
\hline Lapatinib & n.c. & 581.06 & & 6.34 & 0.0029 & 6.302 & 6.21 & 115 \\
\hline Nelfinavir & $50.1(7 \%)^{a}$ & 567.78 & 9.58 & 6.21 & 1.5 & 7.278 & 7.25 & 127 \\
\hline Pibrentasvir & n.c. & 1113.18 & 9.76 & 6.33 & 0.088 & 5.812 & 5.73 & 200 \\
\hline Quinidine & $38.2(8 \%)^{a}$ & 324.42 & 12.8 & 9.28 & 450 & 2.823 & 0.62 & 45.6 \\
\hline Sertraline & $164(8 \%)^{a}$ & 306.23 & & 9.47 & 17 & 5.079 & 2.7 & 12 \\
\hline UCN-01 & $91.4(1 \%)$ & 482.53 & 12.25 & 8.93 & 0.18 & 4.983 & 3.07 & 89.7 \\
\hline Venetoclax & $69.2(32 \%)$ & 868.44 & 4.09 & 8.34 & 0.0072 & 8.051 & 3.97 & 183 \\
\hline
\end{tabular}

n.c., not calculated owing to lack of reference $f_{u} ; \% \mathrm{CV}$, interassay $\% \mathrm{CV}$ calculated from experiments conducted on separated days $(n=2-5$ experiments $)$. Average $\mathrm{P}$ mem $\pm \mathrm{S} . \mathrm{D} .=75 \pm 35 \times 10^{-6}$ $\mathrm{cm} / \mathrm{s}^{2}$.

${ }^{a}$ Intra-assay $\% \mathrm{CV}$ calculated from single experiment run in triplicate. 


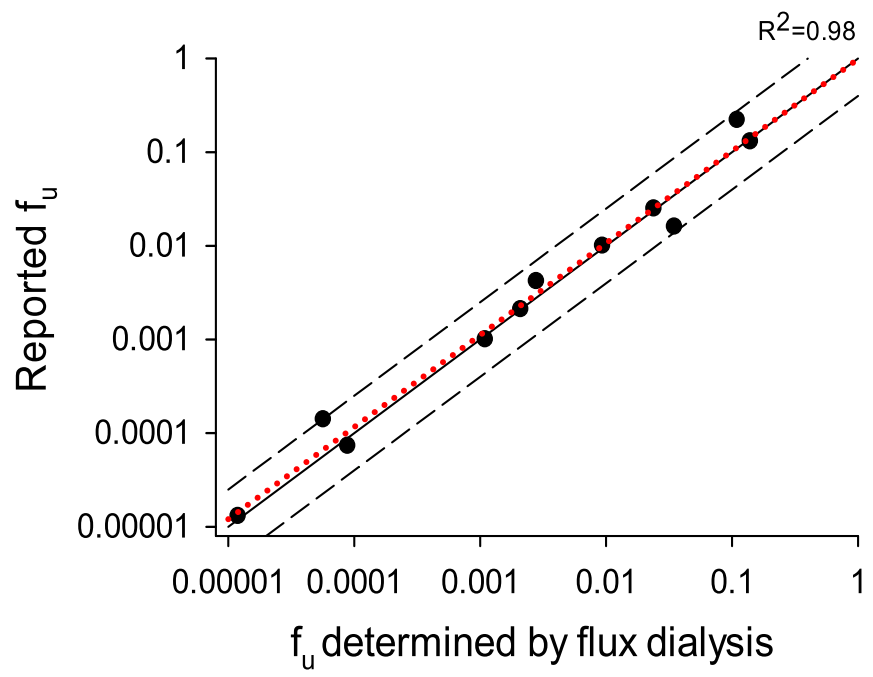

Fig. 3. Unbound fraction determined by flux dialysis versus reported values determined from other methods. Black solid and dashed lines represent the line of unity and \pm 2.5 -fold unity, respectively. Dotted line represents line from log-log orthogonal regression analysis. Flux dialysis $f_{u}$ value for each compound was determined from product each compound's average $R_{\text {slope }}$ and SF (3590 second) according to eq. 8 in the Materials and Methods section. Reported $f_{\mathrm{u}}$ values were obtained from the literature or internal AbbVie reports (glecaprevir and venetoclax).

plasma at clinically relevant concentrations by equilibrium dialysis because receiver buffer concentrations were consistently below the LLOQ. Using the equilibrium dialysis dilution method, venetoclax plasma $f_{\mathrm{u}}$ was measurable in 100 -fold diluted plasma over a compound concentration range from 1 to $30 \mu \mathrm{M}$. No concentration dependency in $f_{\mathrm{u}}$ was observed over the concentration range tested. After correcting for plasma dilution, venetoclax undiluted-plasma $f_{\mathrm{u}}$ value was $0.000013 \pm$ 0.0000064

\section{Discussion}

As early as the 1950s, dynamic dialysis was recognized as a superior approach compared with equilibrium dialysis for measuring the $f_{\mathrm{u}}$ of compounds with high protein binding, but it has been used infrequently, likely because of its technical disadvantages (i.e., requirement for greater radiochemical purity, requirement to estimate $P_{\text {mem }}$, and the need for more sampling time points to define the initial slope of compound appearance). Taking advantage of numerous technological advancements that have been made over the last $\sim 20$ years (improved bioanalytical methods, mass spectrometry, highthroughput 96-well equilibrium dialysis devices, kinetic modeling, etc.), we have tested and validated a modernized variation of dynamic dialysis (i.e., flux dialysis). Kinetic modeling, experimental data, and literature reference data support flux dialysis as a method ideally suited for measuring the $f_{\mathrm{u}}$ of compounds with extremely high protein binding, poor matrix stability, high nonspecific binding, and/or low bioanalytical sensitivity.

The kinetic model used to support the flux dialysis validation and to compare different dialysis-based protein binding methods is similar to a previously described equilibrium dialysis model (Di et al., 2012), but it differs in three ways: 1) the assumption that the binding equilibrium is fast compared with the time scale of dialysis equilibrium $\left(k_{\mathrm{off}}>k_{\mathrm{eq}}\right)$, thus using $f_{\mathrm{u}}$ and $K_{\mathrm{nsb} \text {,device }}$ rather than $k_{\mathrm{on}}$ and $k_{\text {off }}$ parameters; 2 ) saturable protein binding is accounted for through a two-site binding equation rather than a single site; and 3) allowance for dialysis against any matrix rather than conventional buffer only. Neither the Di et al. (2012) nor our model has an explicit algebraic solution for a traditional equilibrium dialysis time course accounting for nonspecific binding or compound degradation; however, the new kinetic model contains a simplified explicit solution when the compound degradation rate is equal in both compartments and when the compound's affinity toward the matrix is greater than its affinity toward the device. This solution is the
A
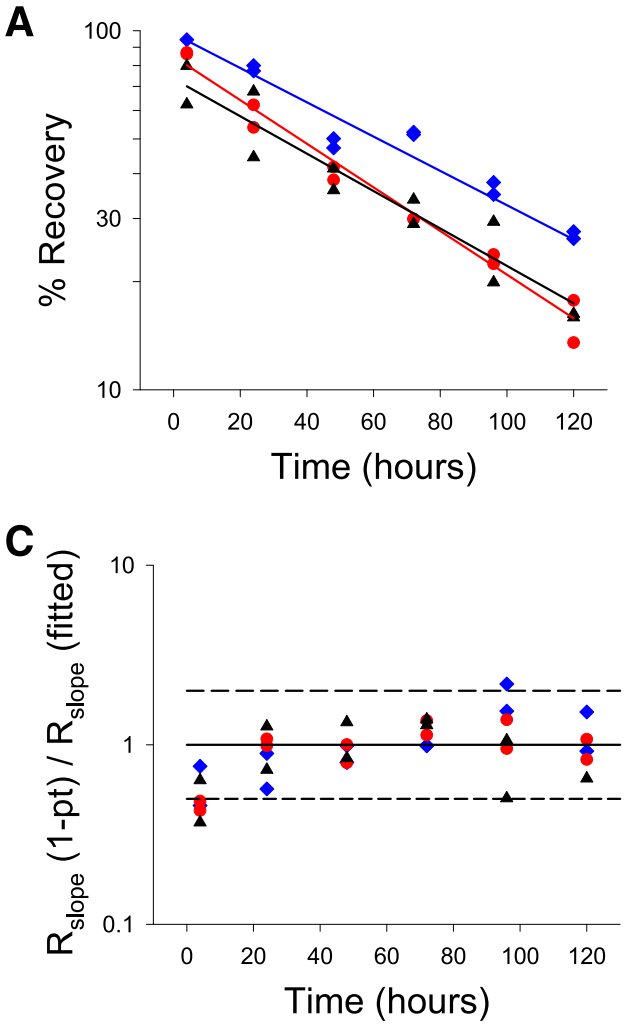

B

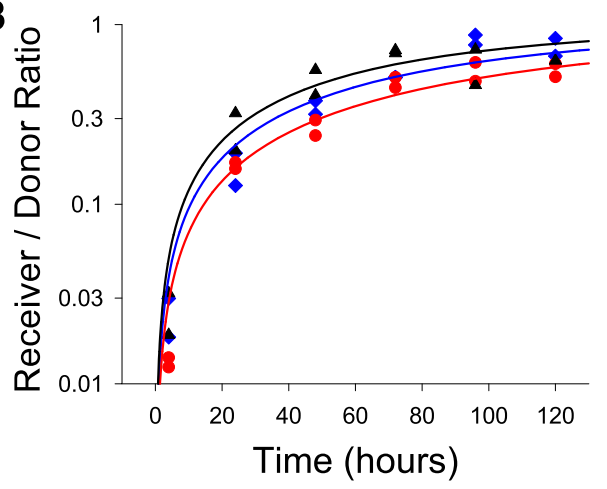

Fig. 4. Indomethacin flux dialysis recovery versus time (A), indomethacin receiver/ donor ratio versus time (B), and concordance between $R_{\text {slope }}$ determined from single time point versus fitted time course (C). Data represent three individual experiments run in duplicate on three separate days with each interday experiment denoted with a different color and shape symbol. Lines in (A) represent nonlinear power-fit function to the $\%$ recovery versus time data from indomethacin flux dialysis experiments. Lines in (B) represent nonlinear fit of $\mathrm{R}=$ $\mathrm{R}_{\mathrm{eq}} \cdot\left(1-\mathrm{e}^{-\mathrm{k} \cdot \mathrm{t}}\right)$ to indomethacin receiver/ donor concentration vs. time data. Solid line in (C) represents the unity line (1.0) and dashed lines represent \pm 2 -fold line of unity (0.5 and 2). $R_{\text {slope }}(1-\mathrm{pt})$ is the $R_{\text {slope }}$ value calculated at each time point using only data from that single time point and eq. $7 . R_{\text {slope }}$ (fitted) is the $R_{\text {slope }}$ value determined from the fit of eq. 5 to the entire receiver/donor concentration time course. 

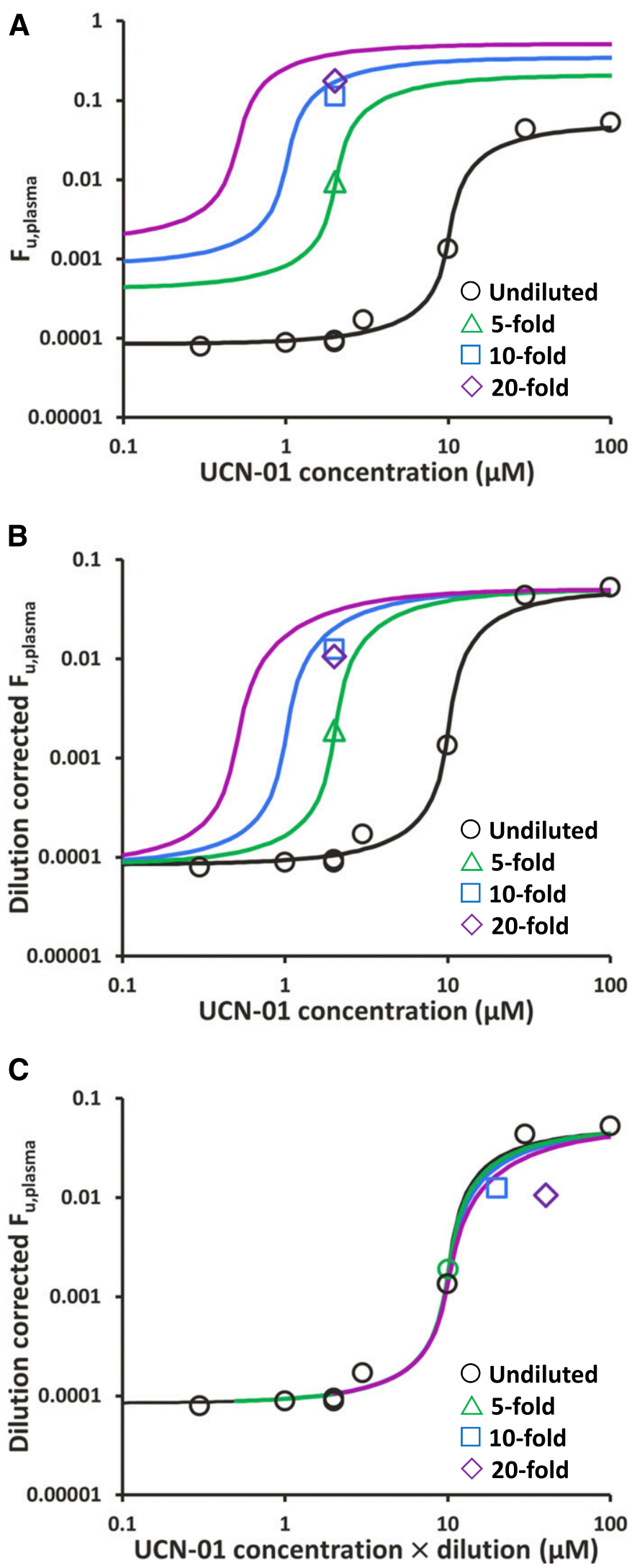

Fig. 5. UCN-01 observed (symbols) and model-fitted (lines) $f_{\mathrm{u}}$ values obtained at various UCN-01 concentrations and plasma dilutions. "Measured" $f_{\mathrm{u}}$ values not corrected for plasma dilution (A), calculated "undiluted" plasma $f_{\mathrm{u}}$ values corrected by eq. 14 for the effect of plasma dilution (B), and calculated "undiluted" plasma $f_{\mathrm{u}}$ values corrected by eq. 14 and by multiplying UCN-01 nominal concentrations by the plasma dilution factor (e.g., $2 \mu \mathrm{M}$ in 5-fold diluted plasma $=10 \mu \mathrm{M}$ ) to account basis for determining $f_{\mathrm{u}}$ from both single and multiple time-point flux data. Another advantage of this new model structure and data treatment is that it is applicable to other dialysis or flux-based experimental approaches (e.g., permeability assays, microdialysis) and could allow more accurate calculations of initial flux rates, permeability, or free drug concentrations (e.g., nanopartical/liposome formulation studies) than commonly used methods that assume initial sink or equilibrium conditions.

The estimated $P_{\text {mem }}$ constant was similar across all compounds tested; allowing the average value of $75.2 \times 10^{-6} \mathrm{~cm} / \mathrm{s}$ to be used for all calculations (i.e., $\mathrm{SF}=3590$ seconds). This value is highly consistent with the permeability through the unstirred water layer determined from four independently published Caco- 2 studies (geomean $P_{\text {appmax }}=73.6 \times$ $10^{-6} \mathrm{~cm} / \mathrm{s}$, range $\left.=37-200\right)($ Avdeef et al., 2005) and the previously reported value for Spectra/Por dialysis tubing $\left(\sim 90 \times 10^{-6} \mathrm{~cm} / \mathrm{s}\right)($ Ross, 1978). The similarity in $P_{\text {mem }}$ constant from this study and the literature indicates that the flux dialysis method could have been comparably validated using an independently determined $P_{\text {mem }}$ constant from the literature (i.e., not using test compounds $f_{\text {u,ref }}$ values to calculate $P_{\text {mem }}$ ) and supports that compound permeability across the unstirred water layer appears to be similar across different in vitro systems and is not significantly influenced by compound properties. A constant $P_{\text {mem }}$ value for all compounds is not unexpected if permeability across membrane unstirred water layer is the rate-limiting step since permeability across the unstirred water layer is compound-independent, assuming the compound in solution behaves as an ideal solution (Wilke and Chang, 1955). The diffusion rate through the unstirred water layer, however, is dependent on the unstirred water layer thickness and temperature (Wilke and Chang, 1955). The $P_{\text {mem }}$ constant could differ depending on experimental conditions (e.g., temperature, shaking speed, membrane thickness), so $P_{\text {mem }}$ should be verified if different experimental conditions are used. Cited literature reports and our own work (data not shown) have shown that shaking speed above specific minima has minimal influence on $P_{\text {mem }}$.

Accurate and precise (i.e., $\leq 2.5$-fold of reported values; median interday $\% \mathrm{CV}=13 \%) f_{\mathrm{u}}$ values for 14 compounds, three with previously unmeasurable $f_{\mathrm{u}}$, were obtained at $1 \mu \mathrm{M}$ concentration in undiluted plasma using the flux dialysis method. Given that 10 of the compounds had $f_{\mathrm{u}} \leq 0.01$ ( $\operatorname{six}$ with $f_{\mathrm{u}}<0.001$ ), this degree of accuracy and precision is particularly striking as traditional protein-binding methods are often validated using few compounds with $f_{\mathrm{u}}<0.01$ or only compare fraction bound values (e.g., fraction-bound values 0.99 and 0.999 are similar, although $f_{\mathrm{u}}$ values differ 10-fold) (Banker et al., 2003; Ye et al., 2017)

Comparison with Traditional Equilibrium Dialysis Method. Equilibrium dialysis is a commonly used and often the preferred method to measure $f_{\mathrm{u}}$; however, $f_{\mathrm{u}}$ results for compounds with high nonspecific binding, matrix instability, and/or poor analytical sensitivity may be prone to inaccuracies. High nonspecific binding to the dialysis device prolongs equilibrium time and can introduce errors if $f_{\mathrm{u}}$ results are determined before equilibrium is reached. Additionally, nonspecific binding to labware may introduce errors in measuring the buffer concentrations, resulting in artificially low $f_{\mathrm{u}}$ values (Di et al., 2012). This is known to be problematic for lipophilic or poorly soluble compounds, such that when sampling buffer from dialysis device, it is recommended that the buffer sample be directly added to the bioanalysis

for the effect of plasma dilution on $\mathrm{C}_{\mathrm{donor}} / \mathrm{B}_{\max }$ ratio (C). Lines represent simultaneous fit of the two-site saturable binding equation, eq. 16, to all UCN-01 $f_{\mathrm{u}}$ data. 
plate containing organic solvent and the pipette tip rinsed by mixing buffer sample and organic solvent together (Kalvass and Maurer, 2002; Di et al., 2012). Flux dialysis mitigates the effects of nonspecific binding since compound is dialyzed against matrix, not buffer (Ross, 1978).

If a compound degrades during equilibrium dialysis, the $f_{\mathrm{u}}$ results may be invalid since the free concentration in buffer and matrix can differ (Di et al., 2012). Depending on the cause of degradation, one or more of the following may overcome this: adding chemical stabilizers/enzyme inhibitors, using heat-inactivated matrix, reducing the temperature, and/or shielding from light. Alternatively, quicker methods, such as ultrafiltration, may be considered. Unfortunately, these "tricks" or alternative methods do not always work for compounds with challenging properties (Di et al., 2017). Flux modeling and experimental results (i.e., indomethacin) show that flux dialysis can be used for unstable compounds as long as the rate of compound loss is equal in both dialysis compartments, as is the case when identical matrix is used on both sides.

Finally, the lowest discrete $f_{\mathrm{u}}$ value measurable by equilibrium dialysis is the buffer bioanalytical LLOQ divided by the total donor concentration. When the buffer concentration at equilibrium is below the LLOQ, only a qualified $f_{\mathrm{u}}$ can be reported (e.g., $<0.01$ ). In contrast, the flux dialysis receiver concentration is always higher than the corresponding free buffer concentrations from equilibrium dialysis since it will approach the total concentration of the donor matrix over time when the matrices are identical.

Comparison with Dilution Method. The dilution method (i.e., using diluted matrix) has been advocated to accelerate equilibrium time and measure $f_{\mathrm{u}}$ for compounds that are too highly bound to measure from undiluted plasma (Riccardi et al., 2015). The flux dialysis method was used to determine $f_{\mathrm{u}}$ values for venetoclax and UCN-01 in undiluted plasma, compounds that could previously be measured only by using the dilution method. The venetoclax $f_{\mathrm{u}}$ showed no concentration dependence and was highly similar between the dilution method and flux dialysis method using undiluted plasma. The similarity in $f_{\mathrm{u}}$ values between the two orthogonal methods provides confidence in the accuracy of the venetoclax $f_{\mathrm{u}}$ value and in the validity of the two methods when $f_{\mathrm{u}}$ is concentrationindependent.

In contrast, UCN-01 $f_{\mathrm{u}}$ values showed large concentration dependence. Even after correcting for the dilution factor, UCN-01 $f_{\mathrm{u}}$ values from diluted plasma in the flux experiment were much larger than those determined from undiluted plasma in the same experiment. The $f_{\mathrm{u}}$ differences between diluted and undiluted plasma were not an artifact of the method, as diluted plasma $f_{\mathrm{u}}$ values from flux closely matched those previously published using equilibrium dialysis with identical compound concentration and plasma dilutions (Riccardi et al., 2015). The apparent $f_{\mathrm{u}}$ differences between the dilution method and undiluted plasma could be reconciled using a two-site saturable binding equation that yielded binding parameters quite similar to previously published values (Fuse et al., 1998). UCN-01 $f_{\mathrm{u}}$ differences obtained using diluted versus undiluted plasma can also be reconciled by a simpler novel approach of multiplying the nominal compound concentrations by the plasma dilution factor. For example, the dilution-corrected $f_{\mathrm{u}}$ value determined from 5-fold diluted plasma at $2 \mu \mathrm{M}$ compound concentrations (i.e., $2 \mu \mathrm{M} \times 5=10 \mu \mathrm{M}$ ) is indistinguishable from the value determined from undiluted plasma at $10 \mu \mathrm{M}$ compound concentration. Consequently, the dilution method offers no apparent advantage over simply using a higher compound concentration in undiluted plasma, and results generated using the dilution method may in fact be misleading for compounds with saturable binding, resulting in an overestimate of $f_{\mathrm{u}}$ if extrapolated to undiluted plasma. Furthermore, based on the dialysis kinetic model, matrix dilution does not meaningfully decrease equilibrium time compared with traditional equilibrium dialysis. Published equilibrium time course for itraconazole, UCN-01, and amiodarone dialyzed in 5-, 10-, and 20-fold diluted plasma against buffer were all parallel at each dilution level, demonstrating that the time to equilibrium does not change with changing $f_{\mathrm{u}}$ or matrix dilution (Riccardi et al., 2015).

Comparison with Competition Method. Equilibrium dialysis of plasma against plasma has been used previously to determine the relative differences in $f_{\mathrm{u}}$ values between different species (Clarke et al., 2008). The method is referred to as the competition method, in which the total concentration ratio between the two different plasmas at equilibrium represents the ratio of $f_{\mathrm{u}}$ between the two plasmas (i.e., equilibrium plasma $_{1} /$ plasma $_{2}$ concentration ratio $=f_{\mathrm{u}, \text { plasma2 }} / f_{\mathrm{u}}$, plasma1). Although measuring total concentration ratio can be technically less challenging than measuring free concentration from buffer, the competition method has two major disadvantages. First, the method does not allow measurements of actual discrete $f_{\mathrm{u}}$ values since only the $f_{\mathrm{u}}$ ratio is determined. Second, equilibrium time may take too long to be practical when plasma ${ }_{1}$ and plasma $_{2} f_{\mathrm{u}}$ values are small (Eriksson et al., 2005). The flux dialysis method exploits the same advantage of the competition method by measuring total plasma concentration rather than free buffer concentration while adding the benefits of allowing for the calculation of actual $f_{\mathrm{u}}$ values and not requiring equilibrium to be reached.

\section{Conclusion}

The modern flux dialysis approach presented here mitigates the disadvantages of previous work using dynamic dialysis. This method uses modern mass spectrometry, high-throughput 96-well equilibrium dialysis devices, and kinetic modeling to eliminate the requirement for greater radiochemical purity and reduces the burden associated with sampling multiple time points under sink conditions. The determination of a constant $P_{\text {mem }}$ has also alleviated the requirement of estimating individual $P_{\text {mem }}$ values. The flux dialysis method enabled discrete measurements of $f_{\mathrm{u}}$ for bedaquiline, lapatinib, pibrentasvir, UCN-01, and venetoclax, five compounds that exhibit extremely high protein binding for which discrete $f_{\mathrm{u}}$ values were not previously measurable from undiluted plasma at $1 \mu \mathrm{M}$ compound concentration. Flux dialysis experiments conducted with identical matrix on both sides offer several advantages compared with other methods, such as increased receiver concentrations (to facilitate bioanalysis), reduced nonspecific binding, valid $f_{\mathrm{u}}$ determination even when the compound is unstable in matrix, no requirement for equilibrium (comparatively shorter dialysis times), and reduced problems associated with buffer samples (e.g., high nonspecific binding, absorption to surfaces, buffer standard curve). Therefore, flux dialysis is recommended as a valid and superior approach to equilibrium dialysis for compounds with high protein binding and other challenging properties.

\section{Appendix A}

The system of differential equations corresponding to the experimental setup in Fig. 1 and the solutions to these equations in various cases of interest are presented below:

Let $A\left(\mathrm{~cm}_{2}\right)$ denote the surface area of the membrane and $P_{\text {mem }}$ $\left(\mathrm{mL} / \mathrm{s} / \mathrm{cm}_{2}\right)$ the unbound membrane permeability to a specific compound. The compartment volumes, $V(\mathrm{~mL})$; fractions unbound, $f_{u}$; total concentrations, $C(\mu \mathrm{M})$; and first-order rates of irreversible loss (e.g., from degradation or covalent binding); $k_{\text {loss }}(1 / \mathrm{s})$, are all specified 
with the appropriate compartment subscript (donor or receiver). The general system is given by eq. A1 and eq. A2:

$$
\begin{aligned}
\frac{d C_{\text {donor }}}{d t}= & \frac{P_{\text {mem }} \times A}{V_{\text {donor }}}\left(f_{\text {u,receiver }} \times C_{\text {receiver }}-f_{u, \text { donor }} \times C_{\text {donor }}\right) \\
& -k_{\text {loss,donor }} \times C_{\text {donor }} \\
\frac{d C_{\text {receiver }}}{d t}= & \frac{P_{\text {mem }} \times A}{V_{\text {receiver }}}\left(f_{u, \text { donor }} \times C_{\text {donor }}-f_{\text {u,receiver }} \times C_{\text {receiver }}\right) \\
& -k_{\text {loss,receiver }} \times C_{\text {receiver }}
\end{aligned}
$$

Introducing permeability-surface area products per unit volume $\mu=P_{\text {mem }} \times A / V$, the preceding equations can be rewritten as eq. A3 and eq. 4A:

$$
\begin{aligned}
\frac{d C_{\text {donor }}}{d t}= & \mu_{\text {donor }}\left(f_{u, \text { receiver }} \times C_{\text {receiver }}-f_{u, \text { donor }} \times C_{\text {donor }}\right) \\
& -k_{\text {loss,donor }} \times C_{\text {donor }} \\
\frac{d C_{\text {receiver }}=}{d t}= & \mu_{\text {receiver }}\left(f_{u, \text { donor }} \times C_{\text {donor }}-f_{u, \text { receiver }} \times C_{\text {receiver }}\right) \\
& -k_{\text {loss, receiver }} \times C_{\text {receiver }}
\end{aligned}
$$

Subject to the initial conditions,

$$
C_{\text {donor }}=\frac{X_{0}}{V_{\text {donor }}}, \quad C_{\text {receiver }}=0,
$$

where $X_{0}$ is the amount of compound added to the donor compartment at time $=0$, analytical solutions for the concentration-time profiles exist and are of the form in eq. A5 and eq. A6:

$$
\begin{gathered}
C_{\text {donor }}(t)=\frac{X_{0}}{V_{\text {donor }}}\left(\frac{1}{r_{2}-r_{1}}\right)\left[\left(r_{2}-\alpha_{\text {donor }}\right) e^{-r_{1} t}+\left(\alpha_{\text {donor }}-r_{1}\right)\right. \\
\left.\quad \times e^{-r_{2} t}\right] \\
C_{\text {receiver }}(t)=\frac{X_{0}}{V_{\text {receiver }}}\left(\frac{f_{u, \text { donor }} \mu_{\text {donor }}}{r_{2}-r_{1}}\right)\left(e^{-r_{1} t}-e^{-r_{2} t}\right)
\end{gathered}
$$

where

$$
r_{1,2}=\left(\alpha_{\text {donor }}+\alpha_{\text {receiver }} \mp \sqrt{\left(\alpha_{\text {donor }}-\alpha_{\text {receiver }}\right)^{2}+4 f_{u, \text { donor }} f_{u, \text { receiver }} \mu_{\text {donor }} \mu_{\text {receiver }}}\right) / 2
$$

$$
\alpha_{\text {compartment }}=k_{\text {loss,compartment }}+f_{u, \text { compartment }} \times \mu_{\text {compartment }} .
$$

These solutions simplify to a manageable form when the assumption of equal loss rates in both compartments is made (i.e., $k_{\text {loss,receiver }}=k_{\text {loss,donor }}=k_{\text {loss }}$ ). In this case,

$$
\begin{aligned}
C_{\text {donor }}(t) & =\frac{X_{0}}{V_{\text {donor }}} \times \frac{1}{(1+\beta)} \times e^{-k_{\text {loss }} t} \times\left(1+\beta e^{-k_{\text {eq }} t}\right) \\
C_{\text {receiver }}(t) & =\frac{X_{0}}{V_{\text {receiver }}} \times \frac{\beta}{(1+\beta)} \times e^{-k_{\text {loss }} t} \times\left(1-e^{-k_{\text {eq }} t}\right),
\end{aligned}
$$

where

$$
\beta=\frac{f_{u, \text { donor }}}{f_{u, \text { receiver }}} \times \frac{V_{\text {receiver }}}{V_{\text {donor }}}
$$

and $k_{e q}$ is the equilibrative rate constant given by eq. A9:

$$
k_{\text {eq }}=\left(P_{\text {mem }} \times A\right)\left(\frac{f_{u, \text { donor }}}{V_{\text {donor }}}+\frac{f_{u, \text { receiver }}}{V_{\text {receiver }}}\right)
$$

The total amount of compound in the system, $A_{\text {total }}$, is not conserved in the system when loss is present. In this simplified case, the total amount of compound exponentially decays and is described by the expression

$$
A_{\text {total }}=\left(C_{\text {receiver }} \times V_{\text {receiver }}\right)+\left(C_{\text {donor }} \times V_{\text {donor }}\right)=X_{0} \times e^{-k_{\text {loss }} t}
$$

The ratio of concentrations,

$$
R=\frac{C_{\text {receiver }}(t)}{C_{\text {donor }}(t)}
$$

from eqs. A7 and A8 yields eq. A10:

$$
R=\frac{f_{u, \text { donor }}}{f_{u, \text { receiver }}} \times \frac{\left(1-e^{-k_{e q} t}\right)}{\left(1+\beta e^{-k_{e q} t}\right)}
$$

and is equal to $f_{u \text {,donor }} / f_{u, \text { receiver }}$ at steady state (time $=$ infinity). This concentration ratio is denoted by

$$
R_{e q}=\frac{f_{u, \text { donor }}}{f_{u, \text { receiver }}}
$$

Note the absence of any impact of $k_{\text {loss }}$ in the equation for $R$ above in eq. A10. This analysis indicates that even in the presence of loss, so long as the loss rates in both compartments are equal, then there is no effect of loss on the concentration ratio.

Assuming equal fraction unbound in both compartments (i.e., $f_{u, \text { receiver }}=f_{u, \text { donor }}=f_{u}$, or equivalently, $R_{\text {eq }}=1$ ),

$$
\begin{gathered}
C_{\text {donor }}(t)=\frac{X_{0}}{V_{\text {receiver }}+V_{\text {donor }}} \times e^{-k_{\text {loss }} t} \\
\quad \times\left(1+\frac{V_{\text {receiver }}}{V_{\text {donor }}} e^{-f_{u}\left(\mu_{\text {donor }}+\mu_{\text {receiver }}\right) t}\right) \\
C_{\text {receiver }}(t)=\frac{X_{0}}{V_{\text {receiver }}+V_{\text {donor }}} \times e^{-k_{\text {loss }} t} \times\left(1-e^{-f_{u}\left(\mu_{\text {donor }}+\mu_{\text {receiver }}\right) t}\right) \\
R=\frac{1-e^{-f_{u}\left(\mu_{\text {donor }}+\mu_{\text {receiver }) t}\right)}}{1+\frac{V_{\text {receiver }}}{V_{\text {donor }}} e^{-f_{u}\left(\mu_{\text {donor }}+\mu_{\text {receiver }}\right) t}}
\end{gathered}
$$

If we further assume that $V_{\text {receiver }}=V_{\text {donor }}=V$ then we obtain the expression

$$
R=\frac{1-e^{-2 f_{u} \mu t}}{1+e^{-2 f_{u} \mu t}},
$$

with a common permeability parameter for each compartment $\mu=P_{\text {mem }} \times A / V$. This expression is equivalent to $R=\tanh \left(f_{u} \mu t\right)$.

If we also consider the case where there is no loss in either compartment (i.e., $k_{\text {loss,receiver }}=k_{\text {loss,donor }}=0$ ), subject to the same initial conditions yields donor and receiver concentration-time equations:

$$
\begin{aligned}
C_{\text {donor }}(t) & =\frac{X_{0}}{V_{\text {donor }}} \times \frac{1}{(1+\beta)}\left(1+\beta e^{-k_{\text {eq }} t}\right) \\
C_{\text {receiver }}(t) & =\frac{X_{0}}{V_{\text {receiver }}} \times \frac{\beta}{(1+\beta)}\left(1-e^{-k_{\text {eq }} t}\right)
\end{aligned}
$$


These equations satisfy the mass conservation equation:

$$
A_{\text {total }}=\left(C_{\text {receiver }} \times V_{\text {receiver }}\right)+\left(C_{\text {donor }} \times V_{\text {donor }}\right)=X_{0},
$$

at all times.

The ratio of concentrations from eqs. A15 and A16 yields the same expression as that achieved when assuming equal loss rates given in eq. A10.

In the case where the concentration ratio is given in eq. A10, then the approximation to the function about time $t=0$, is given by

$$
R_{0} \approx \frac{f_{u, \text { donor }} \times P_{\text {mem }} \times A}{V_{\text {receiver }}} t,
$$

and thus the slope near time $=0$ is given by

$$
R_{\text {slope }}=\frac{f_{u, \text { donor }} \times P_{\text {mem }} \times A}{V_{\text {receiver }}},
$$

which is identical to the expression obtained by assuming initial sink conditions (eq. 4).

To account for nonspecific device binding, we assume that the process is instantaneously in equilibrium, similar to the assumption made for protein binding. In this case, we will need to differentiate between the fraction unbound to the device, $f_{u, \text { device }}$, the fraction unbound to protein, $f_{u, \text { matrix }}$, which is the quantity of interest, and the total composite fraction unbound in the presence of both binding events, $f_{u}$.

If both nonspecific device binding and protein binding processes are in equilibrium, the observed composite fraction unbound in each compartment is given by

$$
f_{u}=\frac{1}{1+\left(\frac{1}{f_{u, \text { matrix }}}-1\right)+\left(\frac{1}{f_{u, \text { device }}}-1\right)}
$$

where

$$
f_{u, \text { device }}=\frac{1}{K_{n s b, \text { device }}+1},
$$

where $K_{n s b \text {,device }}\left(=B_{\max , N S} / K_{D, N S}\right)$ is the nonspecific binding potential to the device under the assumption that $K_{D, N S} \gg C_{u}$. Inserting this expression into the preceding one above gives

$$
f_{u}=\frac{1}{\frac{1}{f_{u, \text { matrix }}}+K_{n s b, \text { device }}}
$$

If nonspecific binding is negligible relative to matrix binding (i.e., $1 / K_{n s b \text {,device }} \gg f_{u, \text { matrix }}$ ), then $f_{u}=f_{u \text {,matrix }}$; however, if nonspecific binding is not negligible, then the expression for $f_{u}$ in eq. A19 must be substituted into eqs. A1-A17. Specifically, the expression for the concentration ratio is

$$
R=R_{e q} \times \frac{\left(1-e^{-k_{e q} t}\right)}{\left(1+\beta e^{-k_{e q} t}\right)},
$$

where

$$
\begin{aligned}
k_{e q}= & \left(P_{\text {mem }} \times A\right)\left(\frac{1}{V_{\text {donor }}\left(\frac{1}{f_{u, \text { donor }}}+K_{\text {nsb,device }}\right)}\right. \\
& \left.+\frac{1}{V_{\text {receiver }}\left(\frac{1}{f_{u, \text { receiver }}}+K_{\text {nsb,device }}\right)}\right) .
\end{aligned}
$$

Notable ramifications of nonspecific binding include the necessity of correcting the $f_{u}$ obtained from the initial slope given by eq. A17 to arrive at the desired $f_{u, \text { matrix }}$. This would require knowledge of nonspecific binding parameters and use of the rearranged form of eq. A19

$$
f_{u, \text { matrix }}=\frac{1}{\frac{1}{f_{u}}-K_{\text {nsb,device }}}
$$

From eq. A20 we observe that in the presence of non-specific binding, the time to equilibrium is extended by a factor of $\left(1+K_{\text {nsb,device }} \times f_{\text {u,matrix }}\right)$.

\section{Appendix B: Fraction Unbound in the Presence of Saturable and Nonsaturable Binding}

In the presence of saturable (S) and nonsaturable (NS) binding sites, the bound concentration at equilibrium is given by the following equation:

$$
C_{b}=\frac{B_{\max } \times C_{u}}{K_{D}+C_{u}}+K_{n s b} \times C_{u}
$$

where $B_{\max }$ and $K_{D}$ are the binding capacity and equilibrium dissociation constant of the specific binding site, and $K_{n s b}\left(=B_{\max , N S} / K_{D, N S}\right)$ is the nonspecific binding potential. The total concentration, $C=C_{b}+C_{u}$, is then

$$
C=\frac{B_{\max } \times C_{u}}{K_{D}+C_{u}}+K_{n s b} \times C_{u}+C_{u} .
$$

Rearranging yields

$$
\left(1+K_{n s b}\right) C_{u 2}+\left(B_{\max }+K_{D}\left(1+K_{n s b}\right)-C\right) \times C_{u}-K_{D} \times C=0
$$

and solving for $C_{u}$ gives

$$
C_{u}=\frac{C-\left(B_{\max }+K_{D}\left(1+K_{n s b}\right)\right)+\sqrt{\left(C-\left(B_{\max }+K_{D}\left(1+K_{n s b}\right)\right)\right)^{2}+4 K_{D}\left(1+K_{n s b}\right) C}}{2\left(1+K_{n s b}\right)} .
$$

The fraction unbound, $f_{u}$, is then given by the ratio of this unbound concentration to the total concentration:

$$
f_{u}=\frac{C-\left(B_{\max }+K_{D}\left(1+K_{n s b}\right)\right)+\sqrt{\left(C-\left(B_{\max }+K_{D}\left(1+K_{n s b}\right)\right)\right)^{2}+4 K_{D}\left(1+K_{n s b}\right) C}}{2\left(1+K_{n s b}\right) C}
$$

\section{Acknowledgments}

We thank David M. Stresser for critically reading the manuscript and editorial comments.

\section{Authorship Contributions}

Participated in research design: Kalvass, Phipps, Jenkins.

Conducted experiments: Stuart, Zhang, Heinle.

Performed data analysis: Kalvass, Phipps.

Wrote or contributed to the writing of the manuscript: Kalvass, Phipps, Jenkins, Nijsen, Fischer, Stuart, Zhang, Heinle.

\section{References}

Avdeef A, Artursson P, Neuhoff S, Lazorova L, Gråsjö J, and Tavelin S (2005) Caco-2 permeability of weakly basic drugs predicted with the double-sink PAMPA pKa(flux) method. Eur $J$ Pharm Sci 24:333-349.

Banker MJ, Clark TH, and Williams JA (2003) Development and validation of a 96-well equilibrium dialysis apparatus for measuring plasma protein binding. J Pharm Sci 92:967-974. Berry LM, Li C, and Zhao Z (2011) Species differences in distribution and prediction of human V (ss) from preclinical data. Drug Metab Dispos 39:2103-2116.

Christensen LK (1959) A method for the determination of free, non-protein bound thyroxine in serum. Scand J Clin Lab Invest 11:326-331. 
Clarke HJ, Gregoire F, Ma F, Martin R, Zhao S, and Lavan BE (2008) Cross-species differential plasma protein binding of MBX-102/JNJ39659100: a novel PPAR-gamma agonist. PPAR Res 2008:465715.

Di L, Breen C, Chambers R, Eckley ST, Fricke R, Ghosh A, Harradine P, Kalvass JC, Ho S, Lee $\mathrm{CA}$, et al. (2017) Industry perspective on contemporary protein-binding methodologies: considerations for regulatory drug-drug interaction and related guidelines on highly bound drugs. $J$ Pharm Sci 106:3442-3452.

Di L, Umland JP, Trapa PE, and Maurer TS (2012) Impact of recovery on fraction unbound using equilibrium dialysis. J Pharm Sci 101:1327-1335.

Eriksson MA, Gabrielsson J, and Nilsson LB (2005) Studies of drug binding to plasma proteins using a variant of equilibrium dialysis. J Pharm Biomed Anal 38:381-389.

Fremstad D, Nilsen OG, Storstein L, Amlie J, and Jacobsen S (1979) Pharmacokinetics of quinidine related to plasma protein binding in man. Eur J Clin Pharmacol 15:187-192.

Fuse E, Tanii H, Kurata N, Kobayashi H, Shimada Y, Tamura T, Sasaki Y, Tanigawara Y, Lush RD, Headlee D, et al. (1998) Unpredicted clinical pharmacology of UCN-01 caused by specific binding to human alpha1-acid glycoprotein. Cancer Res 58:3248-3253.

Indomethacin. (2016) Package insert. Iroko Pharmaceuticals, LLC, Philadelphia.

Kalvass JC and Maurer TS (2002) Influence of nonspecific brain and plasma binding on CNS exposure: implications for rational drug discovery. Biopharm Drug Dispos 23:327-338.

Kalvass JC, Maurer TS, and Pollack GM (2007) Use of plasma and brain unbound fractions to assess the extent of brain distribution of 34 drugs: comparison of unbound concentration ratios to in vivo p-glycoprotein efflux ratios. Drug Metab Dispos 35:660-666.

Meyer MC and Guttman DE (1968) Novel method for studying protein binding. J Pharm Sci 57 $1627-1629$.

Meyer MC and Guttman DE (1970a) Dynamic dialysis as a method for studying protein binding. I. Factors affecting the kinetics of dialysis through a cellophane membrane. $J$ Pharm Sci 59:33-38

Meyer MC and Guttman DE (1970b) Dynamic dialysis as a method for studying protein binding. II. Evaluation of the method with a number of binding systems. J Pharm Sci 59:39-48.
Motoya T, Thevanayagam LN, Blaschke TF, Au S, Stone JA, Jayewardene AL, Chi J, and Aweeka FT (2006) Characterization of nelfinavir binding to plasma proteins and the lack of drug displacement interactions. HIV Med 7:122-128.

Riccardi K, Cawley S, Yates PD, Chang C, Funk C, Niosi M, Lin J, and Di L (2015) Plasma protein binding of challenging compounds. J Pharm Sci 104:2627-2636.

Ronfeld RA, Tremaine LM, and Wilner KD (1997) Pharmacokinetics of sertraline and its $\mathrm{N}$-demethyl metabolite in elderly and young male and female volunteers. Clin Pharmacokinet 32 (Suppl 1):22-30.

Ross HA (1978) A dialysis rate method for the measurement of free iodothyronine and steroid hormones in blood. Experientia 34:538-539.

U.S. Food and Drug Administration (FDA). Center for Drug Evaluation and Research. Sirturo NDA 204384 Clinical Pharmacology and Biopharmaceutics Review, Section 2.2.5.4, December 10, 2012 U.S. Food and Drug Administration. Center for Drug Evaluation and Research. Tykerb NDA 22-059 Clinical Pharmacology and Biopharmaceutics Review, Section 2.2.5.4, January 24, 2007.

Wan H and Rehngren M (2006) High-throughput screening of protein binding by equilibrium dialysis combined with liquid chromatography and mass spectrometry. J Chromatogr A 1102 $125-134$.

Wilke C and Chang P (1955) Correlation of diffusion coefficients in dilute solutions. AIChE J 1: 264-270.

Ye Z, Zetterberg C, and Gao H (2017) Automation of plasma protein binding assay using rapid equilibrium dialysis device and Tecan workstation. J Pharm Biomed Anal 140:210-214.

Address correspondence to: J. Cory Kalvass, Drug Metabolism, Pharmacokinetics and Bioanalysis, Research and Development, AbbVie Inc, Dept. R46V, Bldg. AP9-1, 1 North Waukegan Road, North Chicago, IL 60064-6104. E-mail: j.kalvass@ abbvie.com 